\title{
Event Viewer Design of a Wireless Indoor Sensor Network used in Emergencies
}

\author{
Angel González and W. Velásquez, member IEEE \\ Escuela Superior Politécnica del Litoral, ESPOL, FIEC, P.O. Box 09-01-5863, Guayaquil, Ecuador \\ (ajgonzal, wavelasq)@espol.edu.ec
}

\begin{abstract}
Nowadays, the Internet of Things (IoT) has become a very useful technology to act in situations of danger. This has allowed the implementation of devices composed of multiple sensors, whose purpose is to monitor environments and trigger alerts in an emergency. This document details the process of designing a tool that allows visualizing events derived from the functions of an adapter evacuation management system, which uses sensor systems to monitor different variables in a building. Here, we explain the design decisions made during the development of the platform, the methodology for its implementation and the interaction of the platform with the evacuation system. In addition, a case study is presented in which the platform is tested, using as a model the architecture of the first floor of the administrative building of the Escuela Superior Politécnica del Litoral.
\end{abstract}

Keywords-Platform of visualization; Internet of things; visualization of events; emergency situations; evacuation system;

\section{INTRODUCTION}

Internet of Things (IoT) has opened the development of many applications, due to the popularity in the scientific and the global community [1]. One such application is the emergency area, which seeks to use intercommunicated device networks to safeguard human lives against any event.

Emergency situations require systems to provide reliability and fault tolerance. In the case of event monitoring, it is necessary to make available to the administrator all relevant information, in real time, so that a rapid response can be given to accidents such as fires in a building [2].

At present, the use of environments modeled in 3D is very useful for many applications in fields such as: architecture, urban planning, site monitoring, disaster management and emergencies, among others. They also become a resource that contributes positively to different processes of understanding. 3D environments have been used in urban areas, e.g., to model the infrastructure of a city [3]. Sophisticated applications that allow the reconstruction of disaster areas use architectural and 3D models [4]. Even 3D environments become an important resource when improving the understanding of biological processes [5]. This reveals the importance of visualizations to reinforce the analysis of events [6].

This paper presents the development of a platform that seeks to complement the work proposed by Muñoz et al. [7]. With an event monitoring platform developed using Threejs ${ }^{1}$, WebGL-based library. The platform allows

\footnotetext{
${ }^{1}$ https://threejs.org/
}

the visualization of a 3D model of the first floor of the Administrate building of Escuela Superior Politcnica del Litoral (ESPOL), in whose corridors have been located representations of sensor systems and reactive signaling, which act when receiving data from a server and that allow to visualize different events like: alerts of sensor systems located in the rooms of the building, indicate routes of evacuation in the corridors and present the state of the main exits. The present paper is divided into three sections: Section II describes the methodology. Section III presents the preliminary results with three scenarios. Finally, the paper presents the conclusions about the design and its potential benefits.

\section{Methodology}

In this section, we describe in detail everything related to the implementation of the platform: Choice of Tools, Model Construction, Implementation of events and communication.

The event-viewer has the following requirements: it must be viewed in a web browser; Be interactive and event-driven; The events must be originated upon receiving a bit string from a data source; Should work with acceptable response times and should not incur high processing costs.

\section{A. Tools Selection}

1) Programming Language: Javascript.: Javascript allows the client to observe events such as: clicks, double clicks, mouse pointers, pressed keys, among others; In turn allows the platform to be interactive with the user, allowing the manipulation of the camera in the visualization.

2) Generator Graphics: WebGL and Three.js.: It is necessary to generate a 3D scene with the model of a building in which, in addition, objects will be placed that must interact with the data received. The latter has an independent impact on response times, as it depends on the network, so the platform must generate the graphics without incurring excessive additional overhead that affects the performance of the system.

The WEBGL API [8] works natively in the browser and achieves DOM integration, which allows the use of any language compatible with this interface, i.e., Javascript is perfectly compatible with this API. In addition, WebGL is compatible with all HTML elements and is optimized to 
speed up processing using CPU resources or a graphics card [9].

To reduce complexity in the implementation, a library written in Javascript is used to access the WebGL API. The main advantage of this library is that it significantly reduces the effort required to build an application in which it is necessary to generate graphs "Threejs" [10].

As an alternative to this API, the Unity graphics engine is considered. Unity is an engine oriented to the development of video games in 2D and 3D [11]; One of its main advantages is that it allows to develop a product in a very agile workflow. By its nature, it is event oriented and is a solution applicable to the implementation of threedimensional simulations, for example, in the urban design study [12]. However, to be used in a web application requires the installation of a plugin in the browser and annual licensing paid for professional use.

In spite of allowing a rapid prototyping, the operation of the application developed in Unity in the browser is given through the plugin, which implies that, whenever you want to test the platform and evaluate changes, the application must be built Again and integrated into the browser. On the other hand WebGL with Threjs, to be used by Javascript makes this process easier. Moreover, due to the need for the plugin, additional overhead occurs in response times of the platform.

For the handling of events, due to the needs of the project, WebGL with Threejs allows the client to interact directly with the platform without increasing complexity to the same when using a single language; The communication between the platform and the data source can also be implemented in a simple way.

3) Communication with the platform: Apache ActiveMQ.: Events must be triggered upon receiving a message constructed in the form of a bit string, which in turn comes from a route-mapping algorithm that uses data produced by a sensor system (The implementation of this component is out of the scope of this paper). To cover this requirement, we chose to use a message-oriented Apache ActiveMQ ${ }^{2}$. Apache ActiveMQ offers several advantages over traditional communication schemes (such as the use of databases) and these can be exploited on the platform. It is compatible with Javascript and has an interface to manage a messaging server.

In a traditional communication scheme, the platform should be able to connect to a data server, read a record where a message is stored, delete the record or mark it as read and then trigger the corresponding event as soon as possible. The shortcomings of this scheme for our purpose are that the platform should be responsible not only to process the message, but also to execute additional read and

\footnotetext{
${ }^{2}$ ActiveMQ: http://activemq.apache.org
}

write operations on the server. Moreover, it is necessary that the connection to the data server be maintained over time. This implies that a mechanism must be implemented within the platform that guarantees the persistence of the connection at all times.

The result of implementing these mechanisms, for the handling of messages and the persistence of the connection, is that there is an application that must take care of several tasks incurring additional costs of processing and time. This is where a messaging management middleware is used.

Through the implementation of an Apache ActiveMQ client a persistent communication point between the platform and a message server is created. The connection is constantly evaluated, so that if its loss is detected, it is recovered immediately. The message server is responsible for receiving a string of bits, queuing it and delivering it as soon as possible to the platform. Once delivered the message, this can be stored in a database for the purpose of maintaining a history of events, in a process unrelated to the generation of visualization. The integration of this middleware allows the decoupling of the platform from external processes to the management of events.

\section{B. Construction of the Model}

The model corresponds to a $3 \mathrm{D}$ representation of the first floor of the administrative building of ESPOL. It was built from a 2D plane (Fig. 1) where various aspects of the place are detailed.

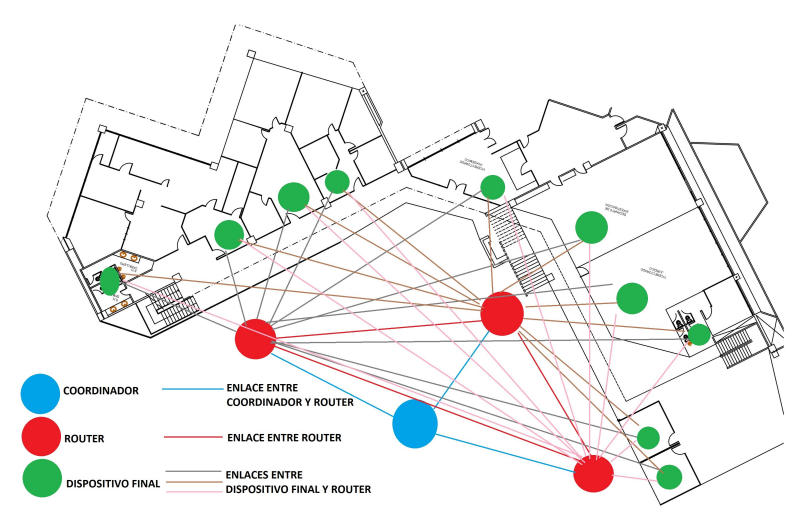

Figure 1. Floor to be designed. It distinguishes the different areas and offices that must be represented in the model

In order to favor the performance of the platform has been divided into sets to the components that make up the model, depending on their function and whether they are interactive or not, so this stage is divided into two parts: Construction of static elements and Construction of Dynamic elements. 
1) Static Elements: The set of static elements is composed of everything that is not interactive in the model. This is: ambient lighting, floors and stairs.

This set of elements is separated from the dynamic elements. It is stored in a JSON file, which is loaded only once when the visualization is started. Objects in this component are not accessible to the functions that control the events.

Stairs represent emergency exits or escape routes. At this stage, Its decided the number of detection devices that would be located in the areas of the model, corresponding to the number of physical devices that would be destined to the real building.

2) Dynamic Elements: The set of dynamic elements is composed of everything that is interactive in the model. This is: Reactive signaling, sensing devices and walls.

The elements in this set are created during the execution of the platform using Javascript code and are used within the functions that control the events.

The reactive signaling is implemented using clusters of 3D objects representing a light system. For the case study, the signs were located in several places of the main aisle and in the emergency exits.

The function of these systems in the corridor is to indicate where to evacuate the area to an emergency situation. This is possible because the lights are arranged in a row that lights up in the direction in which one must travel during an event. For this, the platform receives a string of bits that represents a configuration of an event. This chain is processed by a function and is alerted to each light system, indicating which direction to turn on.

On the other hand, the function of the light systems located in front of the emergency exits is to indicate if it is passable or is disabled for the evacuation. For this, a color code was defined in which the green color indicates that an exit is available for evacuation and the red color indicates that it is disabled during the event. This information is also extracted from the bit string received by the platform.

Sensing Devices are elements that represent a sensor system, which must be located in the actual building. Each of these devices governs one area of the model, so they are dispersed in different places. During this stage the location of each device was defined as well as its effective area of sensing (This effective area is a representation of the sensing range of the devices). As shown in Fig. 2.

The function of these devices is to visually establish in the platform the location of the sensor systems, which remain hidden in the same. These devices reflect different states of real sensing systems. A device may be active during an emergency situation, in which case it sends a signal to trigger an event that indicates that its effective area is compromised during the course of the event.

The walls are part of the set of dynamic elements as they fulfill a function during an event on the platform. This function is to visually delimit the area governed by a sensing

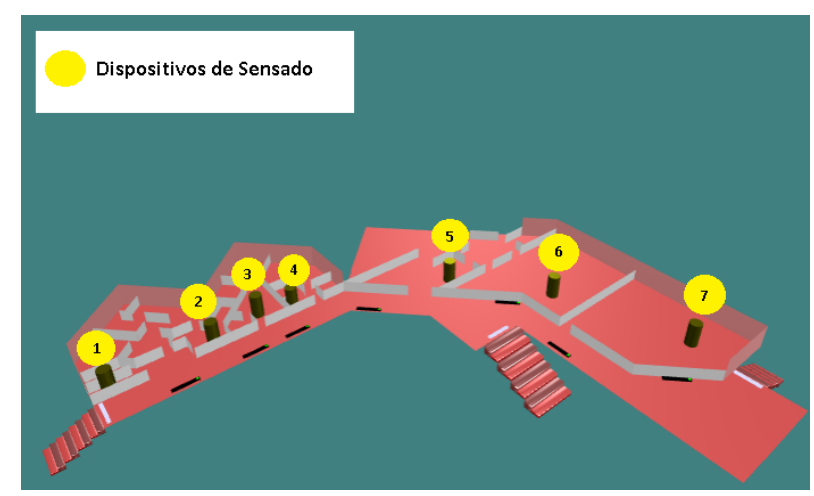

Figure 2. Location of sensing devices in the final model.

device, when an emergency situation has occurred. When a sensing device has received an activity signal, it sends a signal that causes its effective area to blink in a different color than usual.

\section{Events}

At this stage we defined how the platform will be notified about an event to be viewed. For this it was established that all the dynamic elements must act once the bit string representing a configuration is processed. This string represents the result of a route-mapping algorithm and it stores information for each of the components that must interact.

The events implemented are as follows: Turn on reactive signaling in the corridor, turn on reactive signals as emergency exits, notify sensing devices to activate, activate effective areas of the sensing devices. When performing all the individual events in each element, there is a representation of the route of an evacuation route and the visualization of areas compromised in the model during an emergency situation.

\section{Communication}

The use of the Apache ActiveMQ messaging middleware gives the platform a fault-tolerant medium for handling the bit string that should trigger events, with this, a messaging server has been implemented that takes care of receiving the strings and storing them as individual messages in a queue. Meanwhile, in the code of the platform, is embedded a light client that is subscribed to said message queue on the server.

This medium is configured in a way that maintains a persistent communication between the platform and the messaging server, thus ensuring that the platform is always listening to new messages in the event of a new emergency situation.

\section{Preliminary Results}

In order to visualize representations of different emergency situations in the model, several event configurations were established, using different bit strings. 
Several of the configurations present in Table 1, which contain some of the bit strings used during the tests, are described below.

Table I

TEST BIT STRINGS

\begin{tabular}{|c|c|}
\hline Caso & Cadena de Bits \\
\hline $\mathbf{1}$ & 01111010100000010 \\
\hline $\mathbf{2}$ & 11111010101101010 \\
\hline $\mathbf{3}$ & 11111110100010101 \\
\hline
\end{tabular}

In case number one has the following scenario: Sensing device one has detected an emergency situation. No emergency exit is disabled. The signs direct the evacuation to the nearest exits, depending on the area. Although the emergency exit on the left is enabled, note that it is indicated that the evacuation must be executed by the other exits. This is due to the proximity between compromised area and this exit, and with other exits available, it is advisable to avoid the area in danger at all. This case is illustrated in Fig. 3.

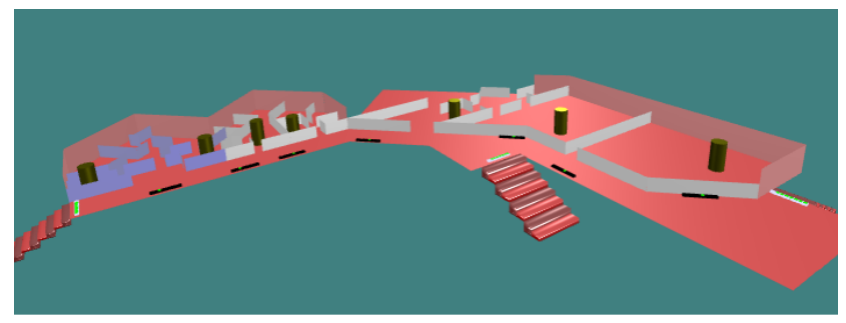

Figure 3. Case Test 1. Notice that only one area has been compromised.

Fig. 4 shows the event of case number two. During this situation, two of the emergency exits have been disabled. This means that there is a risk of transit through these routes and therefore the evacuation must be carried out by the only emergency exit available, which is in the right area of the model. The lighting systems in the corridor are responsible for indicating the route to be followed in order to carry out with the least possible risk of evacuation.

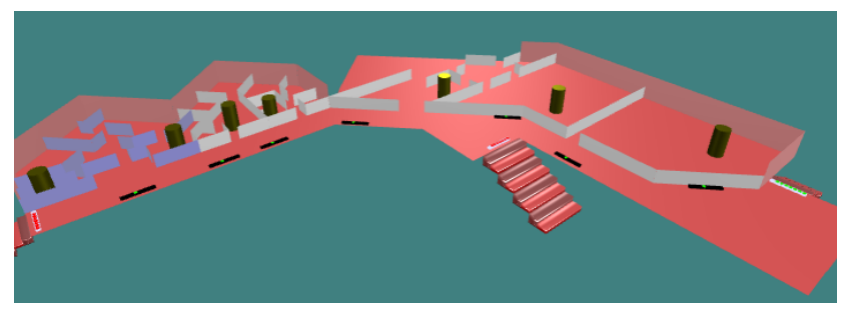

Figure 4. Case Test 2. Both the left and center emergency outputs are blocked.

The third bit string represents a scenario in which several sensing devices have detected danger. Only the emergency exit located in the center of the model is available, so that at both ends of the floor the signs direct the evacuation towards it.

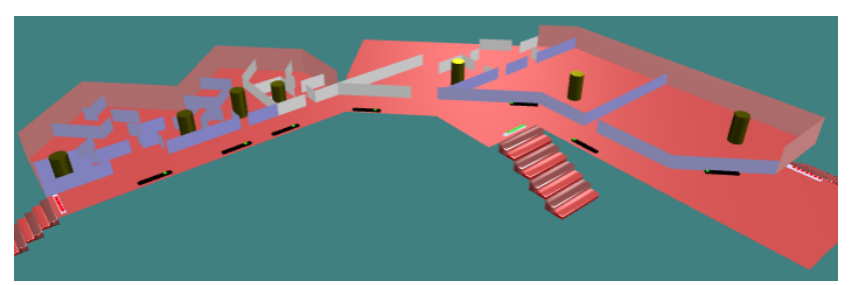

Figure 5. Case test 3. Hazard has been detected in several areas and only one outlet is available.

\section{Conclusions}

In the present work, it was possible to implement a platform to visualize events in emergency situations, in addition to implementing a form of fault tolerant communication. The current model of the platform and its test case can be extended in future works.

Although the platform was evaluated in a local environment, the way in which it was designed allows to assure that it is an implementation oriented to the low consumption of resources. This causes that the execution of the events does not incur high processing costs that add overhead to the running time. Having established this, it is expected that the additional overhead will depend exclusively on how the communication between the platform is configured and an agent that sends the data produced by the algorithm through the Apache ActiveMQ message server. Additional overhead can be produced exclusively by parameters in the network.

In similar implementations, it is suggested that a similar methodology be applied for the construction of the model. This is due to the fact that separating the dynamic components from the static ones aims to reduce the extra processing derived from graphical objects that are not interactive and, therefore, should only be loaded once in the visualization. It is recommended that all static objects be placed within a file (JSON, XML) and loaded together.

The use of a distributed middleware like Apache ActiveMQ can be exploited in a way that gives additional advantages to the platform. An additional utility to integrate into the platform is to configure multiple communications with multiple data sources. It all depends on the approach used to handle the communication that comes from the physical sensors to the platform.

Finally, a tool has been developed that, due to the nature of its implementation, can also be used to perform simulations and visualize the results of path-tracing algorithms. An additional use may be to use the platform as a tool to conveniently locate the sensing devices in the display to determine their ideal locations in the building.

\section{ACKNOWLEDGMENT}

Thanks to the Escuela Superior Politecnica del Litoral (ESPOL) for financing the Kishwar project and the continuity of reactive systems evacuation study in emergencies situations. 


\section{REFERENCES}

[1] Xia, F., Yang, L. T., Wang, L.,\& Vinel, A. (2012). Internet of things. International Journal of Communication Systems, 25(9), 1101.

[2] Maalel, N., Natalizio, E., Bouabdallah, A., Roux, P., \& Kellil, M. (2013, May). Reliability for emergency applications in internet of things. In 2013 IEEE International Conference on Distributed Computing in Sensor Systems (pp. 361-366). IEEE.

[3] Goos, J., Boersma, A. J., Mulder, A., \& Zlatanova, S. (2012, June). 3D Spatial Data Infrastructure. In CESUN 2012: 3rd International Engineering Systems Symposium, Delft University of Technology, The Netherlands, 18-20 June 2012.

[4] Kim, C., Moon, H., \& Lee, W. (2016). Data Management Framework of Drone-Based 3d Model Reconstruction of Disaster Site. ISPRS-International Archives of the Photogrammetry, Remote Sensing and Spatial Information Sciences, 31-33.

[5] Welsher, K., \& Yang, H. (2014). Multi-resolution 3D visualization of the early stages of cellular uptake of peptide-coated nanoparticles. Nature nanotechnology, 9(3), 198-203.

[6] Ali, I., Khan, A. A., Qureshi, S., Umar, M., Haase, D., \& Hijazi, I. (2015). A Hybrid Approach Integrating 3D City Models, Remotely Sensed SAR Data and Interval-Valued Fuzzy Soft Set Based Decision Making for Post Disaster Mapping of Urban Areas. In 3D Geoinformation Science (pp. 87-105). Springer International Publishing.

[7] Muñoz-Arcentales, J. A., Calero, V., Marin-Garcia, I., ChavezBurbano, P., \& Perez-Jimenez, R. (2014, November). Adaptive evacuation management system based on monitoring techniques. In 2014 IEEE Latin-America Conference on Communications (LATINCOM) (pp. 1-5). IEEE.

[8] Tavares, G. (2012). WebGL fundamentals. HTML5 Rocks.

[9] Marrin, C. (2011). Webgl specification. Khronos WebGL Working Group.

[10] Cabello, R. (2010). Three. js. URL: https://github. $\mathrm{com} / \mathrm{mrdoob} / \mathrm{three}$. js.

[11] Engine, U. G. Unity Game Engine-Official Site. Online][Cited: October 9, 2008.] http://unity3d. com.

[12] Indraprastha, A., Shinozaki, M. (2009). The investigation on using Unity3D game engine in urban design study. Journal of ICT Research and Applications, 3(1), 1-18. 